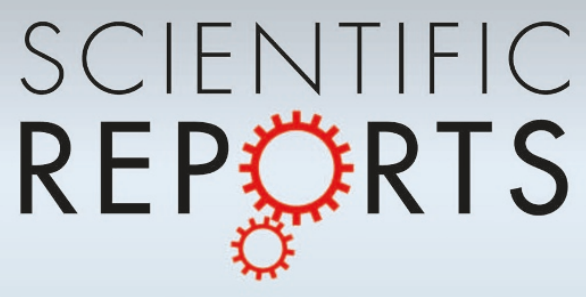

OPEN

\section{SUBJECT AREAS:}

THEORY AND

COMPUTATION

ELECTRONIC PROPERTIES AND MATERIALS

Received

14 February 2014

Accepted

27 May 2014

Published

17 June 2014

Correspondence and requests for materials should be addressed to G.S. (goetz@physik.tucottbus.de)

\title{
Spin excitations of ferronematic order in underdoped cuprate superconductors
}

\author{
G. Seibold', C. Di Castro², M. Grilli² \& J. Lorenzana ${ }^{2}$ \\ ${ }^{1}$ Institut für Physik, BTU Cottbus-Senftenberg, PBox 101344, 03013 Cottbus, Germany, ${ }^{2}$ ISC-CNR, CNISM and Dipartimento di \\ Fisica, Università di Roma "La Sapienza", P.le Aldo Moro 5, 1-00185 Roma, Italy.
}

\begin{abstract}
High-temperature superconductors exhibit a characteristic hourglass-shaped spectrum of magnetic fluctuations which most likely contribute to the pairing glue in the cuprates. Recent neutron scattering experiments in strongly underdoped compounds have revealed a significant low energy anisotropy of these fluctuations which we explain by a model in which topological defects of the antiferromagnet clump to producing domain wall segments with ferronematic order. This state does not invoke global charge order but breaks $C_{4}$ rotational and inversion symmetry. The incommensurability of the low doping charge-disordered state is in good agreement with experiment and interpolates smoothly with the incommensurability of the stripe phase at higher doping. Within linear spin-wave theory the dynamic structure factor is in very good agreement with inelastic neutron scattering data and can account for the observed energy dependent anisotropy.
\end{abstract}

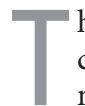
here is emerging a coherent picture concerning the structure of spin excitations in strongly underdoped cuprates (for a recent review cf. e.g. Ref. [1]). In both LSCO (with doping less than $5 \%$ ) $^{2,3}$ and YBCO ${ }^{4-6}$ materials it is now possible to prepare samples with predominantly one twin domain of the orthorhombic structure which allows to elucidate the symmetry of the magnetic excitations in the $\mathrm{CuO}_{2}$ planes. Inelastic neutron scattering (INS) experiments ${ }^{4,7-9}$ reveal a strongly anisotropic low energy spin response which breaks the four-fold symmetry of a $\mathrm{CuO}_{2}$ plaquette. The latter is of course also broken due to the orthorhombic distortion, however, for 'conventional' magnetic excitations this would only induce a small redistribution of weight. In cuprates instead, the low energy response is quasi one-dimensional and corresponds to dynamic incommensurate spin modulations which are oriented along the diagonals of a $\mathrm{CuO}_{2}$ plaquette in $\mathrm{LSCO}$ whereas in $\mathrm{YBCO}$ the response is oriented along the $\mathrm{Cu}-\mathrm{O}$ axis. Upon increasing energy these excitations disperse towards the antiferromagnetic wave-vector $Q_{A F}$ which is reached at a characteristic energy $E_{\text {cross }}$. Above $E_{\text {cross }}$ the magnetic fluctuations more or less isotropically disperse outwards and approach the antiferromagnetic (AF) spectrum of the undoped compound. On the other hand, a scan in the direction orthogonal to the spin modulation reveals a commensurate response up to $E_{\text {cross }}$ whereas for larger energies the excitations start to disperse with almost the same velocity as in the incommensurate modulation direction. Thus the overall magnetic fluctuation spectrum resembles an hourglass parallel to the low energy magnetic spin modulations whereas it is ' $\mathrm{Y}$ '-shaped in the orthogonal direction.

Itinerant approaches ${ }^{10-12}$ can capture the features of hourglass-shaped excitations, however, it is questionable whether these theories are compatible with the strong energy dependent orientational anisotropies described above. In fact, the consideration of spin excitation anisotropies in detwinned $\mathrm{YBa}_{2} \mathrm{Cu}_{3} \mathrm{O}_{6.85}{ }^{13}$ (i.e. close to optimal doping) already requires an anisotropy of $\sim 3 \%$ in the hopping integrals ${ }^{11}$ along the two orthorhombic directions. In contrast, close to the metal-insulator transition orthorhombicity alone cannot account for the strong anisotropies as also revealed by transport experiments on detwinned samples ${ }^{14}$.

The above experimental results are generally well explained by computations of the dynamical structure factor on top of an ordered array of domain walls of the antiferromagnetic order parameter, so-called stripes ${ }^{15-18}$. Not only the hourglass is well described but also general features as, for example, the recovery of $\mathrm{C}_{4}$ symmetry at high energy ${ }^{15}$. On the other hand a severe problem with this interpretation for strongly underdoped cuprates is the apparent absence of charge order. It has been argued that disorder can 'wash out' the stripe charge correlations and corresponding models of disordered (or fluctuating) stripes have in fact been used in order to describe the magnetic hourglass spectra of cuprates and cobalt oxides ${ }^{19,20}$.

Another kind of theories is based on the formation of spin spirals which also break spin rotational invariance similar to stripes, but in the absence of concomitant charge correlations. Early proposals for spirals ${ }^{21}$ have been shown to suffer from an intrinsic instability for homogeneous phases manifested by a negative compressibility ${ }^{22}$. 
On the other hand, holes bound to impurities in the spin glass phase may generate a 'dipolar distortion' in the antiferromagnetic spin background ${ }^{23}$ which in turn can stabilize long-range spiral order ${ }^{24,25}$. Such long-range order coexistent with a disordered background is reminiscent of liquid crystal phases ${ }^{26}$.

Spin excitations for homogeneous (perfectly ordered) helical magnets have been computed within the tJ-model (see e.g. ${ }^{27,28}$ ) and analyzed with regard to the magnetic fluctuations in cuprates ${ }^{29}$. However, to the best of our knowledge detailed computations of the dynamical structure factor for spiral ground states on top of a disordered background to compare with experiment have not yet been done.

More recently, we have proposed a state for heavily underdoped cuprates which shares characteristics of both the dipolar and the stripe scenario. Holes added to the antiferromagnet clump in stripe segments ending in fractional vortex-antivortex (VA) pairs ${ }^{30}$ resembling disclination pairs in nematics ${ }^{26}$. A single short segment produces a dipolar distortion of the AF background similar to Ref. [23]. Under appropriate conditions a finite density of segments globally breaks $C_{4}$ rotational and inversion symmetry and yields spiral long range order, a situation termed ferronematic in Ref. [30]. At large length scales the spin structure coincides with the state proposed in Refs. [24,25], but the microscopic origin of the dipoles, here stemming from the charge segment nematic order, is completely different. In analogy with liquid crystals, at large doping this ferronematic state is expected to transform into a smectic phase thus providing a natural link with the observed stripe state ${ }^{1}$ around doping $1 / 8$. In fact, the ferronematic state has the appealing feature that for parameters appropriate for cuprates the resulting magnetic incommensurability coincides with that obtained for static stripes so that a smooth crossover from the former to the latter with doping is feasible.

It has been shown within Gutzwiller variational calculations of Hubbard-type model $s^{30,31}$ that VA dipoles correspond to stable energy minima for typical cuprate parameters. However, if one is interested only in the spin structure of vortex-antivortex pairs it turns out ${ }^{30}$ that the Gutzwiller results can be reproduced within a spin-only (Heisenberg) model supplemented by frustrating interactions in order to stabilize the segments and the induced ferronematic order. Such a model may appear too simplistic to compute dynamical properties. However, our previous experience with ordered stripes ${ }^{15}$ shows that it can capture many features of a fully fermionic computation, which in the present case would be restricted to too small sizes to be meaningful.

Thus in order to study excitations on top of the ferronematic ground states our investigations are based on the Heisenberg model

$$
H=\sum_{i j} J_{i j} \mathbf{S}_{i} \mathbf{S}_{j}
$$

where the spin structure is taken as coplanar and small out-of-plane tilts due to the Dzyaloshinsky-Moriya interaction are neglected. The segments are modeled as a one-dimensional array of $N_{v a}$ plaquette centered magnetic vortices with alternating topological charge (cf. Fig. 1a). For a number of $N_{\text {seg }}$ segments the total number of holes is given by $N_{h}=N_{v a} N_{s e g} v$ where $v$ denotes the 'filling factor' of a segment. Variational Gutzwiller calculation $s^{30}$ yield $v \approx 0.7$ for diagonal segments.

The following computations are performed for a ratio between next-nearest $\left(J^{\prime}\right)$ to nearest $(J)$-neighbor interaction given by $J^{\prime} / J=$ -0.1 . These parameters are fixed in the undoped phase by the condition that the calculated magnon excitations match the measured spin-wave dispersion ${ }^{32}$.

\section{Results}

We compute the initial spin structure from a linear superposition of randomly positioned vortex-antivortex segments with ferro order as in Ref. [30]
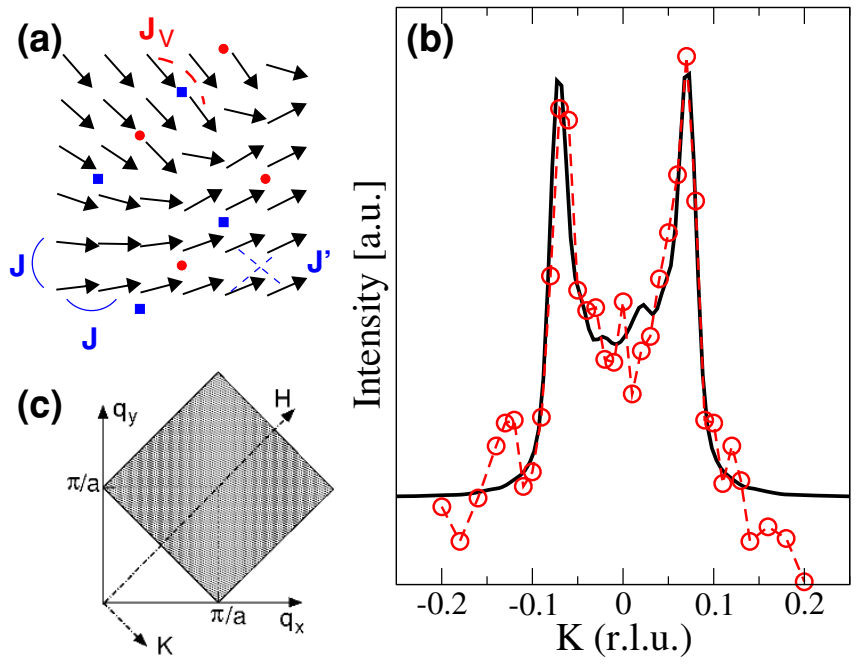

Figure $1 \mid$ Static properties of the ferronematic spin structure. (a) Detail of the staggered ferronematic spin arrangement from a $84 \times 84$ lattice in the $x y$-plane. Segments are build from plaquette centered vortices (squares) and antivortices (circles) which are stabilized by exchange couplings $J_{V}$ between diagonally neighbored spins perpendicular to the vortex-antivortex line. Furthermore a nearest-neighbor $(J)$ and nextnearest neighbor $\left(J^{\prime}\right)$ coupling is implemented in the model. (b) Fit of the spin structure factor (LSCO) for doping $n_{h}=0.05$ and segment length $N_{v a}$ $=4$ perpendicular to the direction of segments. Computations have been done on $84 \times 84$ lattices and we average over 10 segment configurations. The experimental resolution (cf. Fig. 4 of Ref. [2]) has been taken into account by convoluting with a gaussian. Data by courtesy of S. Wakimoto. (c) Sketch of the Brillouin zone geometry used in the present paper. The $K$-direction corresponds to the direction of the disordered spiral modulation and the shaded square indicates the momentum range for the intensity plot of Fig. 3 .

$$
\begin{aligned}
& S^{x}(\mathbf{r})=S_{0} \exp (i \mathbf{Q r}) \cos \Phi(\mathbf{r}) \\
& S^{y}(\mathbf{r})=S_{0} \exp (i \mathbf{Q r}) \sin \Phi(\mathbf{r}) \\
& S^{z}(\mathbf{r})=0 .
\end{aligned}
$$

Here $\mathbf{Q}=(\pi, \pi)$ is the AF wave-vector and

$$
\Phi(\mathbf{r})=\sum_{n=1}^{N_{s e g}} \sum_{i(n)}^{N_{V A}}(-1)^{i(n)} \arctan \left(\frac{x-x_{i(n)}-0.5}{y-y_{i(n)}-0.5}\right)
$$

is the angular spin distortion due to the $N_{\text {seg }}$ segments. In Eq. (3) the sum over $i(n)$ is due to sites belonging to segment $n$ and $(-1)^{i(n)}=$ $1(-1)$ corresponds to a plaquette centered magnetic (anti)vortex. Then the classical energy of Eq. (1) is minimized where the VA structure is stabilized by an additional coupling $J_{V}$ between diagonally neighbored spins across the segments (cf. Fig. 1a). A fit of the VA structure to the result of a Gutzwiller variational computation yields $J_{V} / J=8$ (cf. Methods section). In order to account for fluctuations beyond the variational result we take a smaller value $J_{V} / J=1.5$ which induces the same winding number for the ferronematic spiral as for $J_{V} / J=8$ (cf. supplementary information) and reproduces the low doping magnetic incommensurability of LSCO. Thus this parameter is fixed at the static level and there are no free parameters in the following dynamical computations.

For doping of $n_{h}=0.05$ Fig. $1 \mathrm{~b}$ demonstrates that this approach can reproduce the static spin structure factor of LSCO measured with elastic neutron scattering. Note that the present fit with plaquette centered vortices yields shorter segments with $N_{v a}=4$ as compared to our previous investigations ${ }^{30}$ with site-centered vortex-antivortex pairs. 

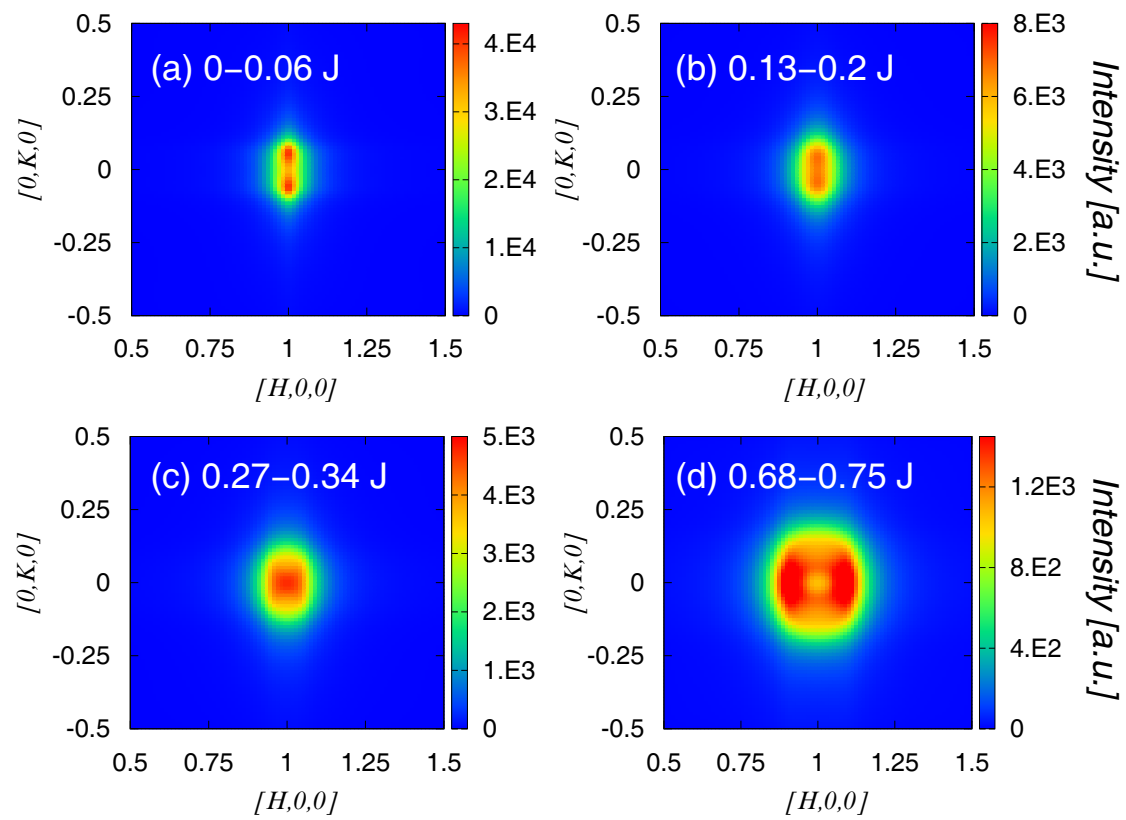

Figure $2 \mid$ Neutron scattering intensity for the ferronematic at doping $\boldsymbol{n}_{\boldsymbol{h}}=\mathbf{0 . 0 5}$. The magnetic neutron-scattering cross section has been integrated over energy ranges (a) $0-0.06 \mathrm{~J}$, (b) $0.13-0.2 J$, (c) $0.27-0.34 \mathrm{~J}$, (d) $0.68-0.75 \mathrm{~J}$. The momentum range corresponds to the shaded area indicated in Fig. $1 \mathrm{c}$. The value of the perpendicular momentum $q_{z}$ has been calculated from energy-momentum conservation for an incident neutron beam energy with $E_{i}=0.3 J$ (panels a,b) and $E_{i}=0.8 \mathrm{~J}$ (panels c,d). Average over 6 configurations.

In order to compute excitations on top of the ferronematic ground state we rotate the spin quantization axis to the direction of the local mean-field magnetization and apply linear spin-wave theory as described in Methods.

It is then straightforward to obtain the spin susceptibilities $\chi_{i j}^{\alpha \beta}(\omega)=\left\langle\left\langle S_{i}^{\alpha} ; S_{j}^{\beta}\right\rangle\right\rangle$ from the equations of motion and to compute the Fourier transformed

$$
\chi_{\mathbf{q}}^{\alpha \beta}(\omega)=\frac{1}{N} \sum_{i j} e^{i \mathbf{q} \cdot\left(\mathbf{R}_{i}-\mathbf{R}_{j}\right)}\left\langle\chi_{i j}^{\alpha \beta}(\omega)\right\rangle_{\text {config. }} .
$$

In practice we evaluate $\chi_{\mathbf{q}}^{\alpha \beta}(\omega)$ for a randomly generated configuration of segments and then take the average over typically 10-20 configurations as indicated by $\langle\ldots\rangle_{\text {config. }}$

The spins of the ferronematic state can fluctuate both in- and outof the $\mathrm{CuO}_{2}$ plane. Both types of excitations contribute to the magnetic neutron-scattering cross section measured by INS which is proportional to

$$
\frac{d^{2} \sigma}{d \Omega d E} \sim \sum_{\alpha \beta} \frac{1}{q^{2}}\left(q^{2} \delta_{\alpha \beta}-q_{\alpha} q_{\beta}\right) \operatorname{Im} \chi^{\alpha \beta}(\mathbf{q}, \omega) \equiv S_{\mathbf{q}}(\omega) .
$$

Here $\chi^{\alpha \beta}(\mathbf{q}, \omega)$ only depends on the in-plane components of $\mathbf{q}$ while the perpendicular component $q_{z}$ enters the cross section only through the prefactors inside the sum. In the extreme case that the $q_{z}$ is much larger than the in-plane components (i.e. for the case that the neutron beam is almost perpendicular to the $\mathrm{CuO}_{2}$ plane and high energies) the response is dominated by the in-plane response $\chi_{\mathbf{q}}^{\|}(\omega)^{33}$. In practice, $q_{z}$ depends on energy and one always has a contribution from perpendicular, in-plane and off-diagonal susceptibilities which we have all included. In our computations we assumed an incoming neutron energy $E_{i}$ and a scattering geometry as in typical neutron scattering experiments (i.e. $q_{x}$ and $q_{y}$ in the first Brillouin zone).

Fig. 2 shows momentum cuts of the neutron scattering intensity within different energy ranges which have been obtained from the same ferronematic configurations for which the static response in Fig. 1b has been evaluated. Momentum cuts along the $H$ - and
$K$-direction for the same energies are reported in Fig. 3. At low energies (panel (a) in Figs. 2, 3) the response is dominated by two incommensurate peaks along the orthorhombic $K$-direction due to the spiral modulation perpendicular to the segments and coinciding in momentum space with the static peaks in Fig. $1 \mathrm{~b}$. The period of the modulation is obtained as $\lambda=2 \pi /\left|q_{K}\right|=14 a_{\text {ortho }}$ corresponding to an incommensurability of $\varepsilon^{\text {ortho }}=1 / 14=0.07143$. Note that for diagonal stripes with $v$ holes per site the incommensurability would be given by $\varepsilon^{\text {ortho }}=n_{h} / v$ thus yielding the same value for $n_{h}=0.05$ and $v=0.7$.

Upon increasing energy the reponse first keeps the elongated shape along the $K$-direction (panel b) but becomes more isotropic at higher energies (panel c). From the momentum cuts in Fig. 3 the
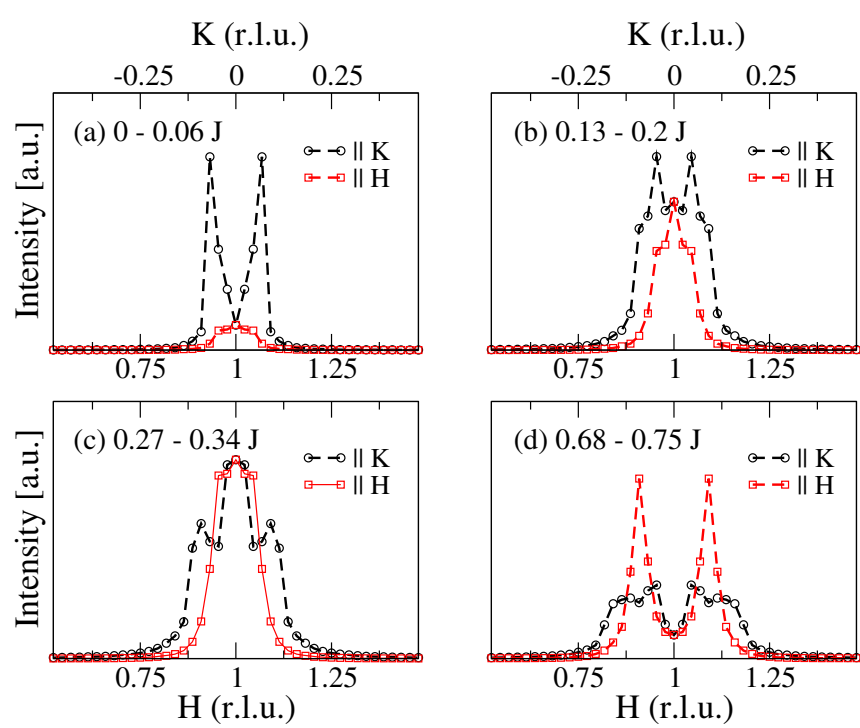

Figure $3 \mid$ Momentum cuts parallel ( $H$-direction, red squares) and perpendicular to the segments ( $K$-direction, black circles) for the intensity distributions shown in Fig. 2. 

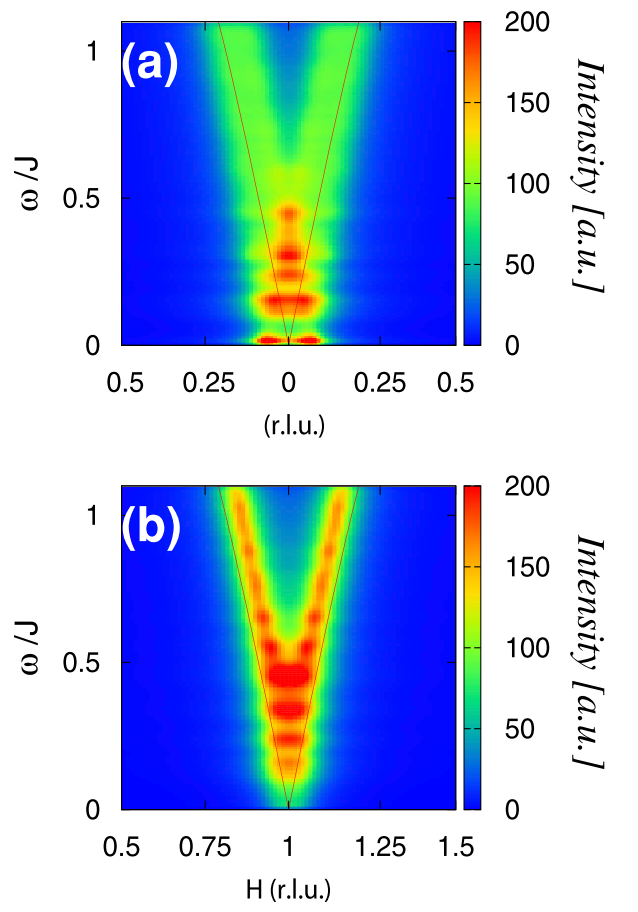

Figure $4 \mid$ Intensity plot of the cross section $\omega S_{\mathrm{q}}(\omega)$ [Eq. (5)] for a momentum cut perpendicular (panel a) and parallel (panel b) to the segments. The value of the perpendicular momentum $q_{z}$ has been calculated from energy-momentum conservation for an incident neutron beam energy with $E_{i}=J$. The solid lines report the magnon dispersion of the undoped antiferromagnet. Average over 6 configurations.

dispersion of excitations along the $K$-direction toward the antiferromagnetic wave-vector can be clearly deduced. Panels b,c of Fig. 3 also reveal a small shoulder in the momentum structure along $K$ which is due to an outwards dispersing branch. Similar to the case of stripes ${ }^{16}$ and enhanced by disorder the corresponding intensity is strongly reduced with respect to the inwards dispersing excitations.

At still higher energies the magnetic scattering intensity acquires a quasi two-dimensional ring-shaped structure (Fig. 2, panel d). The overall features of the intensity distribution are rather similar to data from INS experiments on LSCO (cf. Fig. 1 in Ref. [9]), however, our calculations reveal an anisotropic intensity distribution with variable weight ratio between the $H$ - and $K$-directions at different energy ranges. (cf. panel (c) of Fig. 3). This feature resembles an analogous rotation of weight observed in the magnetic scattering from static stripes in $\mathrm{La}_{1.875} \mathrm{Ba}_{0.125} \mathrm{CuO}_{4}{ }^{34}$, whereas the data from strongly underdoped LSCO seem to indicate a more isotropic distribution of weight. We should remark that for the case of stripes we observe that a computation in a spin only model (as here) yields a more anisotropic response than a more accurate computation in a fermion mode ${ }^{15}$. Thus we attribute this small difference in anisotropy to the simplicity of the model. Unfortunately a fermion model could not be used due to the large size required to take into account disorder with high momentum resolution.

The magnetic dispersions along $K$ and $H$ directions can be deduced from Fig. 4 which shows the corresponding cuts of the magnetic structure factor Eq. (5) weighted by energy. Panel (a) of Fig. 4 clearly reveals the basic features of the hourglass spectrum although the resolution is limited due to the large periodicity of the ferronematic spiral as compared to the size of the lattice which can be dealed with. The large intensity feature corresponds to the inwards dispersing magnetic excitations which merge at $Q_{A F}$ and subsequently disperse outwards again with reduced intensity. At high energies the magnetic fluctuations approach the magnon dispersion

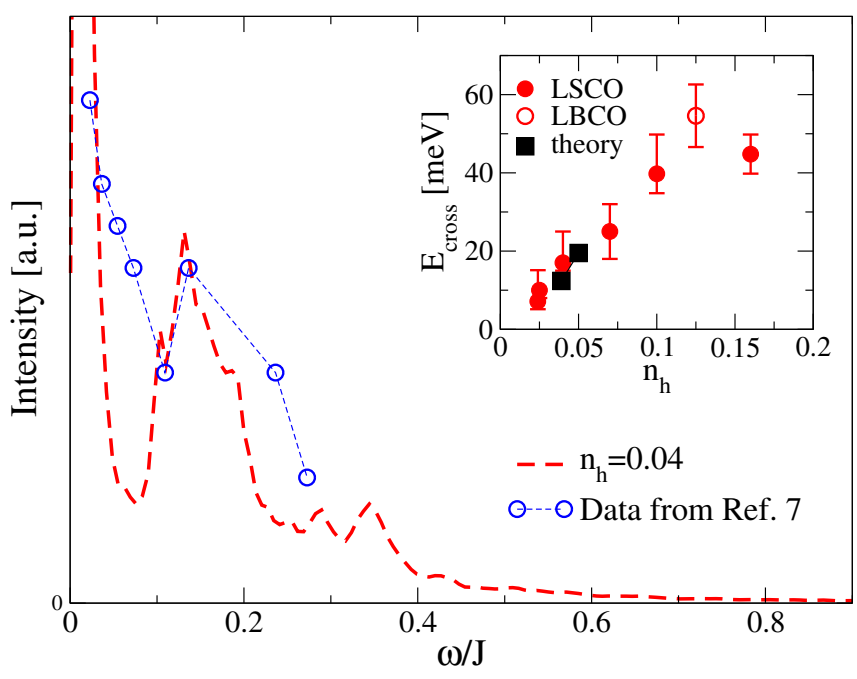

Figure $5 \mid$ Energy dependence of the magnetic cross section $S_{\mathrm{q}=Q_{A F}}(\omega)$ (cf. Eq. (5)) at the antiferromagnetic wave-vector. The main panel reports $S_{\mathrm{q}=Q_{A F}}(\omega)$ for doping $x=0.04$ (dashed) together with experimental data extracted from Fig. 1 of Ref. [7]. Inset: Comparison of the crossing energy with experimental data for LSCO (solid circles) and LBCO (open circle) from Ref. [8]. For the conversion into energy units we use $J=112 \mathrm{meV}^{32}$ and a frequency independent renormalization factor $Z_{c}=1.18^{35}$.

of the undoped antiferromagnet which is indicated by the solid line in Fig. 4. The Y-shaped structure of the spin excitations in the parallel direction to the segments is reported in panel (b) of Fig. 4. Here the low energy magnetic excitations are confined to $Q_{A F}$ but also start to follow those of the undoped AF above the crossing energy $E_{\text {cross }}$.

The location of $E_{\text {cross }}$ is difficult to determine from Fig. 4 due to the limited resolution. Instead we deduce this scale from the energy dependence of the magnetic structure factor for the antiferromagnetic wave vector $Q_{A F}$ which is shown in Fig. 5 for doping $x=0.04$. Since the ferronematic spin spiral is strongly disordered the static structure factor also displays small but finite weight at $Q_{A F}$. This induces a 'quasi-elastic' peak at $\omega \rightarrow 0$ in the frequency dependent magnetic cross section, $S_{\mathrm{q}=Q_{A F}}(\omega)$ in addition to the higher energy feature at $E_{\text {cross }} \approx 0.14 \mathrm{~J}$. We can convert the 'J-scale' into energy units by taking $J=112 \mathrm{meV}^{32}$ and a frequency independent renormalization factor $Z_{c}=1.18^{35}$ which yields good agreement with the magnon dispersion of the undoped AF. Based on this conversion we also show in Fig. 5 experimental $S_{\mathrm{q}=Q_{A F}}(\omega)$ data for $\mathrm{La}_{1.96} \mathrm{SrO}_{0.04} \mathrm{CuO}_{4}$ which we have extracted from Fig. 1 of Ref. [7]. Interestingly also these data support an increase of $S_{\mathrm{q}=Q_{A F}}(\omega)$ for $\omega$ $\rightarrow 0$ in agreement with the persistence of AF domains in an otherwise incommensurate ordered spin system. Finally, the inset to Fig. 5 reports $E_{\text {cross }}$ for $x=0.04$ and $x=0.05$ which fits rather well into the experimentally determined ${ }^{8}$ evolution of $E_{\text {cross }}$ with doping. In contrast, stripe calculations in the strongly underdoped regime usually significantly overestimate $E_{\text {cross }}{ }^{17}$ although this may also be cured by disorder ${ }^{19}$.

\section{Discussion}

We have shown that the spectrum of spin excitations computed on top of a ferronematic ground state is in qualitative and quantitative agreement with inelastic neutron scattering experiments on cuprate superconductors in the spin glass phase. Since the ferronematic also can be thought of arising from melted stripes our theory provides an explanation for the continuous evolution of the hourglass-shaped magnetic excitations from low to optimal doping. It should be mentioned that preliminary Monte-Carlo simulations also seem to 
a) GA
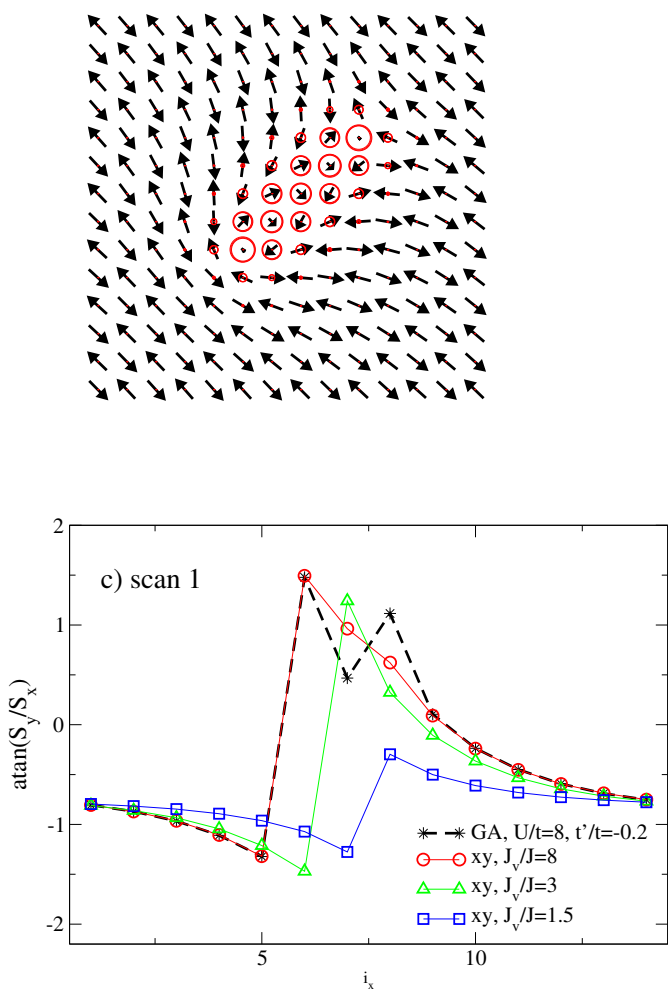

b) xy
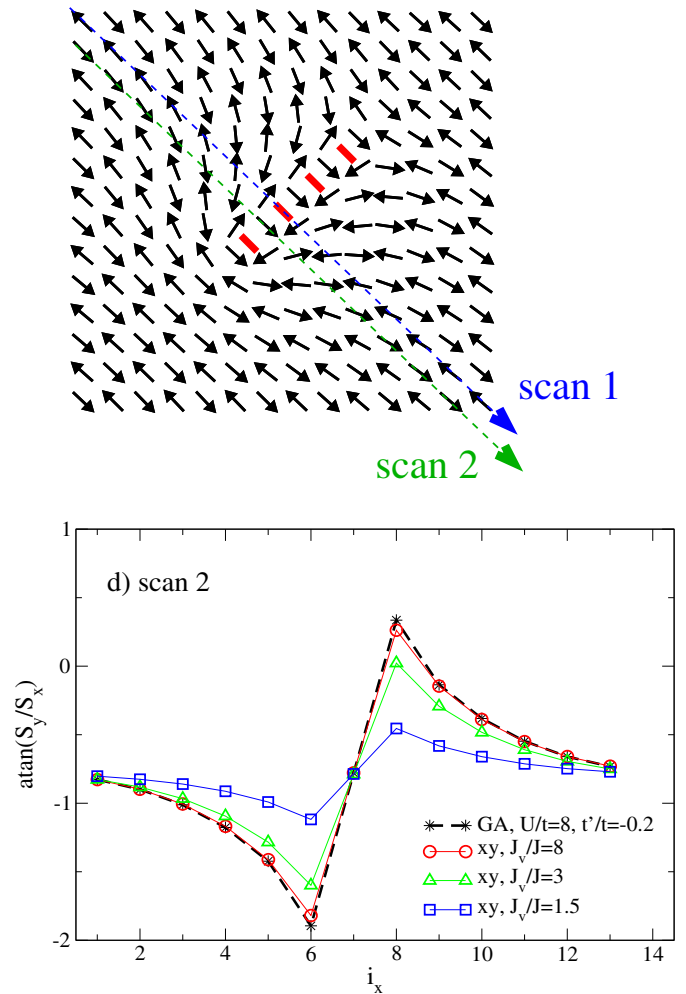

Figure 6 Comparison of spin textures obtained from the Hubbard model within the GA (a) and the Heisenberg model (b) The GA solution shown in panel (a) has been obtained for 4 holes in a $14 \times 14$ system. Parameters: $U / t=8, t^{\prime} / t=-0.2$. The spin structure of panel (b) results from the minimization of the Heisenberg model. Here the 4 diagonal exchange couplings which are indicated by bars are set to $J_{v} / J=8$ and all other nearestneighbor couplings are $J^{\prime} / J=-0.1$. Panels (c), (d) compare the angular rotation of the spins $\left(=\operatorname{atan}\left(S_{y} / S_{x}\right)\right)$ along the scans indicated in panel $(\mathrm{b})$.

support smectic charge correlations which are stabilized by longrange Coulomb interactions. While such correlations between disordered stripes can also induce an incommensurate magnetic response $^{19}$, the magnetic fluctuations of the ferronematic spiral are due to the disordered magnetic background which is induced by the vortex and antivortex dipoles of the charge segments.

Experimentally, the ferronematic state is supported by the observation of a low temperature ferroelectric phase in the spin glass phase of cuprates ${ }^{36}$. In fact, the ferronematic state breaks inversion symmetry so that on general grounds ${ }^{37}$ a ferroelectric distortion becomes possible e.g. via the inverse Dzyaloshinskii-Moriya mechanism ${ }^{38}$. The 'smoking gun' experiment would then be to test whether the onset temperature of incommensurate spin fluctuations coincides with that of the ferroelectric as predicted by our model.

\section{Methods}

Parameters for the effective Heisenberg model. In order to estimate the effective coupling $J_{v}$ across the plaquette centered magnetic vortices and antivortices (cf. Fig. 1) we perform a variational calculation based on the single-band Hubbard model

$$
H=\sum_{i j, \sigma} t_{i j} c_{i, \sigma}^{\dagger} c_{j, \sigma}+U \sum_{i} n_{i, \uparrow} n_{i, \downarrow}
$$

including nearest-neighbor $(t)$ and next-nearest neighbor $\left(t^{\prime}\right)$ hopping. Ground state properties are obtained from the Gutzwiller variational wave-function $\left\langle\Psi_{G}\left|=\hat{P}_{G}\right| \Phi_{0}\right\rangle$ where $\hat{P}_{G}=\Pi_{i}\left[1-\left(1-g_{i}\right) n_{i, \uparrow} n_{i, \downarrow}\right]$ partially projects out doubly occupied sites from the Slater determinant $\left|\Phi_{0}\right\rangle$. We evaluate $E_{G}\left(\left\{g_{i}\right\}\right)=\left\langle\Psi_{G}|H| \Psi_{G}\right\rangle\left\langle\left\langle\Psi_{G} \mid \Psi_{G}\right\rangle\right.$ in the limit of infinite dimensions ${ }^{39}$ corresponding to the so-called Gutzwiller approximation (GA) and minimize with respect to the variational parameters $g_{i}$. We set $U / t=8$ and $t^{\prime} / t=-0.2$ which within a time-dependent extension of the GA reproduces the spinexcitations of undoped LSCO $\mathrm{LS}^{15,16}$

Panel (a) of Fig. 6 reports the spin/charge texture obtained in this way for 4 holes (192 particles) on a $14 \times 14$ lattice with periodic boundary conditions. Hole charges (indicated by circles) are arranged in $2 \times 2$ plaquettes aligned along the diagonal direction. The center of the plaquettes alternately contains a magnetic vortex and antivortex, respectively, inducing a phase change of the $\mathrm{AF}$ order across the segment. This feature can be modeled within the Heisenberg model by diagonal couplings $J_{v}$ indicated by solid bars in panel (b) of Fig. 6. Panels (c,d) compare the angular spin twist $\sim \operatorname{atan}\left(S_{y} / S_{x}\right)$ between GA computation and minimization of the Heisenberg model energy along the two scans indicated in panel (b). We find that for $J_{v} / J=8$ there is excellent agreement of the spin far field (which is determined by the dipole strength of the segment) between both approaches. On the other hand, one may argue that the variational GA overestimates the localization of hole charges and that including corrections beyond the GA would lead to actually smaller values of $J_{v} / J$. The phase change across the segment is then reduced for smaller $J_{v} / J$ as can be seen from panels (c,d). However, as long as $J_{v} / J \gtrsim 1.5$ we find that the static and dynamic spin structure factor only weakly depends on $J_{v} / J$ as demonstrated in the supplementary material where we show results for the GA fitted value $J_{y} / J=8$. In the 'Results' section instead we have taken a smaller value $J_{v} / J=1.5$ in order to include fluctuations beyond the GA variational approach.

Evaluation of spin excitations. Spin excitations on top of the ferronematic ground state are obtained by applying a rotation to the spins

$$
S_{i}^{x}=\tilde{S}_{i}^{x} \cos \left(\Theta_{i}\right)+\tilde{S}_{i}^{y} \sin \left(\Theta_{i}\right)
$$

$$
S_{i}^{y}=\tilde{S}_{i}^{y} \cos \left(\Theta_{i}\right)+\tilde{S}_{i}^{x} \sin \left(\Theta_{i}\right)
$$

$$
S_{i}^{z}=\tilde{S}_{i}^{z}
$$

so that the spin quantization axis points in the direction of the local mean-field magnetization ${ }^{40}$.

For this effective ferromagnetic state one can apply the Holstein-Primakoff transformation:

$$
\tilde{S}_{i}^{x}=\frac{1}{2}\left(a_{i}^{\dagger}+a_{i}\right)
$$




$$
\begin{gathered}
\tilde{S}_{i}^{y}=-\frac{1}{2}+a_{i}^{\dagger} a_{i} \\
\tilde{S}_{i}^{z}=\frac{1}{2 i}\left(a_{i}^{\dagger}-a_{i}\right) .
\end{gathered}
$$

The linear-spin wave theory Hamiltonian reads,

$$
H=\sum_{i j} A_{i j} a_{i}^{\dagger} a_{j}+\frac{1}{2} \sum_{i j} B_{i j}\left[a_{i}^{\dagger} a_{j}^{\dagger}+a_{j} a_{i}\right]
$$

with

$$
\begin{gathered}
A_{i i}=-\sum_{j} J_{i j} \cos \left(\Theta_{i}-\Theta_{j}\right) \\
A_{i \neq j}=\frac{1}{2} J_{i j}\left[\cos \left(\Theta_{i}-\Theta_{j}\right)+1\right] \\
B_{i j}=\frac{1}{2} J_{i j}\left[\cos \left(\Theta_{i}-\Theta_{j}\right)-1\right]
\end{gathered}
$$

where $\Theta_{i}$ is the angle the spin $i$ forms with the $x$-axis. Here we used the fact that the configurations are coplanar. The absence of unstable spin-wave modes in our analysis supports this assumption.

1. Fujita, M. et al. Progress in Neutron Scattering Studies of Spin Excitations in HighTc Cuprates. J. Phys. Soc. Jpn. 81, 011007 (2012).

2. Wakimoto, S. et al. Direct observation of a one-dimensional static spin modulation in insulating $\mathrm{La}_{1.95} \mathrm{Sr}_{0.05} \mathrm{CuO}_{4}$. Phys. Rev. B 61, 3699 (2000).

3. Matsuda, M. et al. Static and dynamic spin correlations in the spin-glass phase of slightly doped $\mathrm{La}_{2-x} \mathrm{Sr}_{x} \mathrm{CuO}_{4}$. Phys. Rev. B 62, 9148 (2000).

4. Hinkov, V. et al. Electronic Liquid Crystal State in the High-Temperature Superconductor $\mathrm{YBa}_{2} \mathrm{Cu}_{3} \mathrm{O}_{6.45}$. Science 319, 597 (2008).

5. Haug, D. et al. Neutron scattering study of the magnetic phase diagram of underdoped $\mathrm{YBa}_{2} \mathrm{Cu}_{3} \mathrm{O}_{6+x}$. New J. Phys., 12, 105006 (2010).

6. Daou, R. et al. Broken rotational symmetry in the pseudogap phase of a high-Tc superconductor. Nature 463, 519 (2010).

7. Matsuda, M. et al. Magnetic Dispersion of the Diagonal Incommensurate Phase in Lightly Doped $\mathrm{La}_{2 x} \mathrm{Sr}_{x} \mathrm{CuO}_{4}$. Phys. Rev. Lett. 101, 197001 (2008).

8. Matsuda, M., Fernandez-Baca, J. A., Fujita, M., Yamada, K. \& Tranquada, J. M. Detailed structure of the low-energy magnetic dispersion of the diagonal incommensurate phase in $\mathrm{La}_{1.975} \mathrm{Sr}_{0.025} \mathrm{CuO}_{4}$. Phys. Rev. B 84, 104524 (2011).

9. Matsuda, M., Granroth, G. E., Fujita, M., Yamada, K. \& Tranquada, J. M. Energydependent crossover from anisotropic to isotropic magnetic dispersion in lightly doped $\mathrm{La}_{1.96} \mathrm{Sr}_{0.04} \mathrm{CuO}_{4}$. Phys. Rev. B 87, 054508 (2013).

10. Norman, M. R. Relation of neutron incommensurability to electronic structure in high-temperature superconductors. Phys. Rev. B 61, 14751 (2000).

11. Eremin, I. \& Manske, D. Fermi-Liquid-Based Theory for the In-Plane Magnetic Anisotropy in Untwinned High-Tc Superconductors. Phys. Rev. Lett. 94, 067006 (2005).

12. Sherman, A. \& Schreiber, M. Low-frequency incommensurate magnetic response in strongly correlated systems. Phys. Rev. B 69, 100505 (2004).

13. Hinkov, V. et al. Two-dimensional geometry of spin excitations in the hightransition-temperature superconductor $\mathrm{YBa}_{2} \mathrm{Cu}_{3} \mathrm{O}_{6+x}$. Nature 430, 650 (2004).

14. Ando, Y., Segawa, K., Komiya, S. \& Lavrov, A. N. Electrical Resistivity Anisotropy from Self-Organized One Dimensionality in High-Temperature Superconductors. Phys. Rev. Lett. 88, 137005 (2002).

15. Seibold, G. \& Lorenzana, J. Doping dependence of spin excitations in the stripe phase of high-Tc superconductors. Phys. Rev. B 73, 144515 (2006).

16. Seibold, G. \& Lorenzana, J. Magnetic Fluctuations of Stripes in the High Temperature Cuprate Superconductors. Phys. Rev. Lett. 94, 107006 (2005).

17. Seibold, G. \& Lorenzana, J. Calculation of incommensurability and spin excitations of diagonal stripes in underdoped lanthanum cuprates. Phys. Rev. B 80, 012509 (2009).

18. Seibold, G., Markiewicz, R. S. \& Lorenzana, J. Spin canting as a result of the competition between stripes and spirals in cuprates. Phys. Rev. B 83, 205108 (2011).

19. Vojta, M., Vojta, T. \& Kaul, R. K. Spin Excitations in Fluctuating Stripe Phases of Doped Cuprate Superconductors. Phys. Rev. Lett. 97, 097001 (2006).
20. Andrade, E. C. \& Vojta, M. Disorder, Cluster Spin Glass, and Hourglass Spectra in Striped Magnetic Insulators. Phys. Rev. Lett. 109, 147201 (2012).

21. Shraiman, B. I. \& Siggia, E. D. Spiral phase of a doped quantum antiferromagnet. Phys. Rev. Lett. 62, 1564 (1989).

22. Auerbach, A. \& Larson, B. E. Doped antiferromagnet: The instability of homogeneous magnetic phases. Phys. Rev. B 43, 7800 (1991).

23. Aharony, A., Birgeneau, R. J., Coniglio, A., Kastner, M. A. \& Stanley, H. E. Magnetic phase diagram and magnetic pairing in doped $\mathrm{La}_{2} \mathrm{CuO}_{4}$. Phys. Rev. Lett. 60, 1330 (1988).

24. Hasselmann, N., Castro Neto, A. H. \& Morais Smith, C. Topological defects and the spin glass phase of cuprates. Europhys. Lett. 56, 870 (2001).

25. Sushkov, O. P. \& Kotov, V. N. Theory of Incommensurate Magnetic Correlations Across the Insulator-Superconductor Transition of Underdoped $\mathrm{La}_{2} \mathrm{Sr}_{x} \mathrm{CuO}_{4}$. Phys. Rev. Lett. 94, 097005 (2005).

26. Chaikin, P. M. \& Lubensky, T. C. Principles of Condensed Matter Physics. Cambridge University Press, Cambridge, 1995.

27. Gan, J., Andrei, N. \& Coleman, P. Spin waves in a doped antiferromagnet. J. Phys.: Condens. Matter 3, 3537 (1991).

28. Shraiman, B. I. \& Siggia, E. D. Excitation spectrum of the spiral state of a doped antiferromagnet. Phys. Rev. B 46, 8305 (1992).

29. Milstein, A. I. \& Sushkov, O. P. Effective field theories and spin-wave excitations in helical magnets. Phys. Rev. B 84, 195138 (2011).

30. Seibold, G., Capati, M., Di Castro, C., Grilli, M. \& Lorenzana, J. Hidden ferronematic order in underdoped cuprates. Phys. Rev. B 87, 035138 (2013).

31. Seibold, G. Vortex, Skyrmion, and elliptical domain-wall textures in the twodimensional Hubbard model. Phys. Rev. B 58, 15520 (1998).

32. Coldea, R. et al. Spin Waves and Electronic Interactions in $\mathrm{La}_{2} \mathrm{CuO}_{4}$. Phys. Rev. Lett. 86, 5377 (2001).

33. Lorenzana, J., Seibold, G. \& Coldea, R. Sum rules and missing spectral weight in magnetic neutron scattering in the cuprates. Phys. Rev. B 72, 224511 (2005).

34. Tranquada, J. M. et al. Quantum magnetic excitations from stripes in copper oxide superconductors. Nature 429, 534 (2004).

35. Singh, R. R. P. Thermodynamic parameters of the $T=0$, spin-1/2 square-lattice Heisenberg antiferromagnet. Phys. Rev. B 39, 9760 (1989).

36. Viskadourakis, Z. et al. Low-temperature ferroelectric phase and magnetoelectric coupling in underdoped $\mathrm{La}_{2} \mathrm{CuO}_{4+x}$. Phys. Rev. B 85, 214502 (2012).

37. Mostovoy, M. Ferroelectricity in Spiral Magnets. Phys. Rev. Lett. 96, 067601 (2006).

38. Sergienko, I. A. \& Dagotto, E. Role of the Dzyaloshinskii-Moriya interaction in multiferroic perovskites. Phys. Rev. B 73, 094434 (2006).

39. Gebhard, F. Gutzwiller correlated wave functions in finite dimensions d: A systematic expansion in 1/d. Phys. Rev. B 41, 9452 (1990).

40. Merino, J., McKenzie, Ross, H., Marston, J. B. \& Chung, C. H. The Heisenberg antiferromagnet on an anisotropic triangular lattice: linear spin-wave theory. J. Phys.: Condens. Matter 11, 2965 (1999).

\section{Acknowledgments}

We are grateful to John Tranquada for a critical reading of the manuscript and for providing to us detailed information on the scattering conditions of time-of flight neutron scattering experiments with direct geometry instruments. G.S. acknowledges support from the Deutsche Forschungsgemeinschaft. J. L. is supported by the Italian Institute of Technology through seed project NEWDFESCM.

\section{Author contributions}

G.S. developed the numerical code and wrote the manuscript; G.S., C.D.C., M.G. and J.L. contributed to analyze the results and write the manuscript.

\section{Additional information}

Supplementary information accompanies this paper at http://www.nature.com/ scientificreports

Competing financial interests: The authors declare no competing financial interests.

How to cite this article: Seibold, G., Di Castro, C., Grilli, M. \& Lorenzana, J. Spin excitations of ferronematic order in underdoped cuprate superconductors. Sci. Rep. 4, 5319; DOI:10.1038/srep05319 (2014).

This work is licensed under a Creative Commons Attribution-NonCommercialShareAlike 4.0 International License. The images or other third party material in this article are included in the article's Creative Commons license, unless indicated otherwise in the credit line; if the material is not included under the Creative Commons license, users will need to obtain permission from the license holder in order to reproduce the material. To view a copy of this license, visit http:// creativecommons.org/licenses/by-nc-sa/4.0/ 